\title{
THE 'PATH' THEORY OF GORTIGAL FUNGTION.*
}

\author{
BY
}

\author{
W. R. ASHBY, LeAYESDEN.
}

There have been few general theories to account for the functioning of the central nervous system, but there has been one which, although it has never been put forward with any definite evidence, nevertheless has gradually grown until it remains practically the only theory in the field. I refer to the "path' theory, which states, roughly, that the functions of the central nervous system are controlled by chains of neurons laid down as a path so as to conduct the impulse to its appropriate end-organ: that the paths are strictly constant, thus accounting for the fact that the reflexes and reactions are largely constant : that learning consists of the opening up of new paths: and that memory consists of the retraversing of some old path by another impulse.

But it is beginning to become apparent that the theory is in need of a thorough overhaul, especially as it has never been clearly stated but tends to lie in the background, emerging to explain some convenient fact yet not being questioned when facts appear which it is powerless to explain. It appears either explicitly or implicitly in neurology, in physiology, and particularly in any work which attempts to deal with physiological psychology : and, curiously enough, it is particularly in the latter that it breaks down. It will be shown that there are certain facts which are incompatible with this theory ; but first it is as well to examine its beginnings and its development ${ }^{1}$ since this throws some light on its present position.

Galen, about A.D. 190, first showed that an excised muscle may be made to twitch by pinching the nerve. He found that pinching the nerve at any point of its course resulted in contraction, and that division of the nerve between the point of application of the stimulus and the muscle prevented contraction. Here, then, was the first 'path' theory, a theory which was obviously justified by the facts. It was assumed that something passed from the point of stimulation to the muscle and it was found that, given the state of the path, one could predict whether or not the muscle would contract.

From this point there was a gap, but by 1800 the grosser anatomy of the nervous system was well known, though practically nothing was known of its physiology. In 1811, Bell ${ }^{2}$ made his fundamental discovery of the functions

* Submitted for publication January. 1931. Since this article was handed to the Editor, Professor K. S. Lashley has, in lectures given at King's ('ollege, London, developed a theory similar to that described here. This paper was completed before their delivery.-W. R. A. 
of the anterior and posterior spinal roots. It was found that in any reflex the impulse always went in at the posterior roots and always emerged from the anterior roots to go to the muscles. Further it was found that a break in any part of this circuit always abolished the reflex. At this time nothing was known of the histology of the spinal cord and it was naturally imagined that the course of the impulse across the spinal cord was determined by a direct pathway through the cord in the same way as it was determined by the peripheral nerves. These early workers, in fact, imagined that the afferent and efferent nerves were merely two halves of one nerve which ran direct from the skin through the spinal cord to the muscle. Thus in 1822 Bell $^{3}$ developed his idea of a "nervous circle,' and in 1850 Marshall Hall ' first used the term ' reflex arc.'

At about this time, the microscope was first being used, and the histology of the central nervous system was being investigated. The discovery of the multicellular nature of the spinal cord at first disturbed these ideas, but the postulate of a 'fixed path' was already well established from the work done on peripheral nerves : and a chain of nerve-cells was soon postulated in place of the old concept of one continuous nerve from skin to muscle. Since the peripheral path was fixed, it was quite naturally assumed that the path through the spinal cord would also be fixed and immutable. But the theory of a fixed path was postulated, not because the evidence showed that it must be fixed but merely because it was impossible to construct any reasonable theory which allowed the path to vary. It must be remembered that at that time practically the only thing known about nerves was that they conducted impulses. The simple theory that the reflex was controlled and produced by a predetermined path without branches which led straight from skin to muscle through the spinal cord was easy to understand and fitted the few facts quite well.

Lewes $^{5}$ in 1867 was among the first to criticise this theory, and his criticism holds good to-day. He attacked the evidence for the existence of the anatomical structures which are assumed to underlie reflex action. He pointed out that it is a case of 'an imaginary physiology based on an imaginary anatomy.' To quote his words :- "The sensory nerve is not seen to enter the spinal cord and to pass over along a particular path to a corresponding point of exit. It is seen to enter the gray substance, which is continuous throughout the spinal cord : it is there lost to vieu, its course being untraceable" (my italics). He concludes that it is the physiology of the central nervous system which controls the reflexes, and not its macroscopic or microscopic anatomy.

Since then, the neurologists in particular have been mapping out tract after tract in the nervous system, until the conclusion seems obviously to be that 'all is tracts.' But there is a tendency to forget that all these tracts are white matter tracts and that, as far as is known, no single gray matter tract has ever been discovered. It is not permissible to ignore the essential difference between the gray and the white matter. The two are fundamentally 
different, and analogies from the one to the other are not permissible. The gray matter is the essential substance of the central nervous system, and it is in this substance that all the purposive, integrative functions of the nervous system are worked out. But the body and brain are large, and the gray matter is not sufficient to extend all over the body ; so, to connect up the masses of gray matter with one another and with the muscles, glands, etc., a highly specialised connecting tissue is developed - the white matter, a tissue apparently devoid of all functions save that of conduction. The white matter is obviously constructed as a 'path' system, but this does not entitle us to draw the conclusion that the gray matter will also be built on the same plan, since the functions of the two types are so different.

The 'path' theory of gray matter function, originated and developed by neurologists in their work on the conduction tracts of the nervous system, has slowly crept in, almost without being noticed. The standard text-book diagram of a spinal reflex, for instance, begs the question at the outset with its picture of afferent and efferent fibres, which are known to be immutable, shown connected together by a single cell, thereby suggesting strongly to all who read that the whole mechanism is known to be that of a path, and that this path could be dissected out if only our technique were good enough. Herrick writes of these diagrams as follows:-_" The simplified neurologic schemata which we use as pædagogic devices are necessary instruments for unlocking those doors of the student's mind which lead into the more recondite secrets of nervous organization. But having opened the door in this way, the key should be laid aside and not regarded as even an adequate symbol of the actual organization, as is so often done in both clinical and psychological practice."

The text-book diagram, having 'explained' the mechanism of reflex action, is succeeded by other diagrams showing how summation, inhibition, etc., may also be explained in terms of paths. From this it is but a short step to the explanation of all nervous activity by means of 'permanent path' mechanisms. Association is assumed to consist of the linking up of nervecells ; memory is explained as the re-threading of an old path by a fresh impulse, and it is added that, of course, the passage of an impulse lowers the resistance of the synapses, and so on. But examples need not be multiplied. Lashley $^{7}$ in a recent paper has pointed out that many of the current ' explanations' have no basis in fact. To take one definite instance-the idea that the passage of an impulse lowers the resistance of a synapse-there is no evidence, clinical or experimental, for this idea, yet it is met with repeatedly.

There is one other ' fact ' which must be mentioned since unless it is refuted it is apt to be used as a proof of the path theory. I refer to the assumption of the constancy of the path of the impulses in a simple reflex. It is usually assumed, almost axiomatically, that the path followed by the impulses in a simple reflex such as the corneal is constant in its passage from neuron to neuron. It must be pointed out that this is not a fact but a hypothesis-a 
hypothesis supported, incidentally, by little or no evidence. Whether the impulses take the same path each time or whether they take a variable path must at present be considered an open question.

There is a second fault in the diagram type of explanation. It lies in the supposition that a reaction may be broken down into its individual impulses and that the reaction may be adequately examined by examining the paths of the individual impulses. This, although a common idea, cannot be entertained. Many impulses come up the same nerve-fibre many times a dayconsider for instance one of the sensory nerves of the leg. But in each case, what happens to the impulse depends on what other impulses' say' about the situation. It is fairly clear that the cerebral cortex reacts to stimuli not as if they were a mass of independent stimuli but as an interconnected and integrated bunch. If one may use an analogy for purposes of illustration, one may compare the original individual impulses to the pieces of a jig-saw puzzle. The cortex does not merely react to each piece of the puzzle, it assembles them into a connected picture, which is obviously something quite different from the arithmetical sum of the individual pieces. Having assembled the picture, the brain then reacts to the picture as a whole. This, of eourse, is really only pointing out anew that the function of the brain is integrative, and not merely summative. The methods of explanation which deal with isolated stimuli are therefore fallacious.

After these preliminary remarks we may pass to the actual clinical and experimental evidence which is against the path theory of cortical function.

The extent to which the nervous system is independent of localized injuries has been strikingly demonstrated by Lashley ${ }^{8.910}$, who has been working on the maze-habit in rats, and its relation to cortical injury. He took a number of rats and taught them a given maze. He then operated on them and destroved various parts of the cortex, removing different parts in different rats, so that altogether he had removed any given part of the cortex in at least one rat. When they had recovered he put them in the maze again, to see how much they had altered. He found that any part of the cortex could learn the maze-habit, and that no part of the cortex showed any superiority over any other part. Frontal, visual, parietal cortices, all were just as capable of learning the maze-habit as any other part. On testing them in mazes of varying difficulty, he found that the rat's ability to learn a difficult maze depended solely on the amount of cortex left behind, again not depending in the least on the position of the cortex left.

From this and from the rest of his work he $e^{8.9}$ has developed the concept of the 'equipotentiality of the cortex,' meaning by that that all parts are equivalent for learning, that the cortex acts as a whole in any given reaction, and that its ability depends almost entirely on the amount of cortex available. The position of the lesion appears to be without significance. "The cortex, after an injury, promptly readjusts itself, but in virtue of its lowered mass, 
at a lower level." $\mathrm{He}^{\text {? }}$ ends with the following conclusion :-- "Such phenomena suggest that the nervous system is capable of a self-regulation which gives a coherent logical character to its functioning no matter how its anatomical constituents may be disturbed." Such a conclusion is clearly against the rigorously anatomical path theory.

These results are very similar to those obtained by Pavlov ${ }^{11}$ in his attempts to localise the conditioned reflex in the cortex. He took many dogs, established a conditioned reflex in them and then tried to destroy the reflex by incisions through the cortex. These incisions should, on the path theory, cut through the path if the cuts were sufficiently numerous. He found, like Lashley, that the conditioned reflex seemed to have no definite localisation in the cortex, although he had previously shown conclusively that the conditioned reflex depends upon, and uses, the cerebral cortex.

Such results as. these are clearly against the path theory. The cortex evidently does not consist of a number of fixed paths, one to each reaction.

Similar evidence against the theory is furnished by observation of brain injuries in man, such as by tumour, etc. Injury to the cortex will obviously destroy certain nerve-cells and their connections. On the path theory we should expect that the patient would lose those reactions which depended on the paths which have been cut, and would retain all others. In practice, this sharp-cut loss of reactions is not seen in the majority of cases. The patient, for instance, does not forget his name while remembering his address : nor does he remember his aunt's name while forgetting that of his dog. On the contrary, the disturbance shown seems to be like that observed by Lashley in rats, viz., that there is a general lowering of intelligence, so that all simple things tend to be remembered while all complicated matters tend to be forgotten. This state is quite different from the condition to be expected from the path theory, where we would have expected a complete loss of a number of specific reactions, side by side with complete normality in other reactions, the result in any given reaction depending on whether the path for the reaction was cut or not.

Of a different type but of similar import are the experiments of Marina ${ }^{12}$. He crossed the internal and external recti muscles of the eye on one side in apes and found that, as soon as the wounds were healed, the eyes moved normally and together. According to the path theory, inversely conjugate movements should have ensued since the paths were crossed. If it is objected that the nerve centres almost instantly recrossed the paths, one may justly ask why should a path once firmly laid down ever bother to recross itself ? The path theory suggests that the eyes would probably go on moving contrariwise indefinitely, for it offers no reason as to why the paths should ever be altered once they are laid down, especially since the continuous passage of impulses should have worn the path into a deep rut. From this and from many other similar experiments Marina concluded that the conduction pathways have no predestined function. He demanded a new foundation for the physiology of the brain. 
There is yet a third type of experiment which shows how impotent the path theory is to account for the facts.

To explain the phenomenon of the subsequent recognition of objects once seen, the obvious explanation according to the path theory is that the object, by stimulating a particular group of cells in the retina, thereby stimulates a particular group of cells in the visual cortex. Should this group be stimulated again in the same way, the similar excitation resulting is ' recognised' by the cortex as being the same as previously and the object itself is thereby recognised.

But this idea, however simple it may appear at first, soon breaks down when one considers the various ways in which the object may vary its projection on the retina without the faculty of recognition being upset. For instance, I may see an object in one part of the visual field and note its appearance. Later I may catch sight of the same object in another part of the visual field altogether, but I recognise it at once, and without any difficulty, in spite of the fact that the object is now stimulating a group of cells, not one of which uas necessarily concerned in the original seeing. It is obvious that the path theory breaks down under these circumstances. There is, in fact, no end to the transformations which an object may undergo without affecting the ability to recognise it. These transformations (of position) all have the effect of stimulating quite a different group of nerve-cells in the retina and visual cortex, without having any effect on the ability to recognise the object. Thus the object may first be placed vertically; it is then placed on its side and re-inspected-it is recognised at once. It may be brought nearer the eye, thus increasing the area over which it stimulates the retina. It may be moved away until it is near the limit of definition. It may be reversed by viewing it in a mirror so that its mirror-image is seen. All day long in everyday life we are continuously recognising things that we have never before seen in exactly that position In all these transformations, the object in its second position may stimulate a group of cells in the retina and visual cortex quite different from those stimulated on the first viewing, yet the object is recognised at once.

From these results we can see that the recognition of an object is independent of the cells it stimulates. It is the pattern which counts, not the position.

What, then, are we to substitute for the path theory?

I propose here to sketch out a possible alternative theory, not so much to suggest that it is definitely the actual state of affairs in the nervous system, but chiefly in order to demonstrate practically that there are alternative theories, since there seems to be a general impression that there is no alternative theory - that the nervous system must, a priori, work on a 'path' mechanism. On the contrary, the path theory has no such a priori validity. That there is at least one alternative theory will be shown below.

Let us consider first the question of the equivalence of the different images thrown on the visual cortex by a given object. We saw above that it is the 
pattern that counts, not the position of the pattern. We have to ask what type of mechanism would produce this result.

A mechanism of this type exists in nature. Consider a pond being affected by ripples. We may suppose the pond to have in it a rod which taps the water at certain intervals. Around the rod would develop a system of ripples, forming a definite pattern. If the rod continued to tap the water in the same way, then the pattern formed would remain constant as long as the tapping continued. In the simple case of a single rod it would, of course, form a series of concentric circles. Now stop the tapping, allow the water to settle down, and then recommence the same tapping at another part of the pond-the same system of wave-patterns will again be formed. Here, then, we have a mechanism which shows the features desired ; we have a permanent pattern which is independent of the localisation of the pattern. The wave-system may be more complex without in the least altering the fundamental features. The tapping may be done by a number of rods, all going at different speeds. In this case, of course, the pattern produced will be complex and will exhibit not merely waves but also interference phenomena, but nevertheless, if the same set of rods beating in the same way be started in another part of the pond, then the pattern will develop in just the same way again. This pattern is quite independent of its localisation in the pond, and its nature will depend solely on the number, position and frequency of the stimuli (rods) producing it. It may, however, be affected by the water in certain ways. Firstly, if the water is at all 'patchy' in its nature, say in temperature, then the pattern will not work itself out in the same way in different places, but the pattern produced will depend upon the distributior, of temperatures in the two areas. Further, if the water be changed uniformly, say by being changed to treacle, then the pattern will change, but in this case it will merely suffer a uniform change by becoming smaller.

The case of ripples on water has been considered because it is simple and well-known. Actually there are many such systems known, all of which show the same fundamental properties, viz., that there is a fixed pattern movable over a material substratum in such a way that the form of the pattern is independent of the position of the pattern. In all such cases, the nature of the pattern developed depends upon (a) the timing, nature, etc., of the rods producing it, and $(b)$ on the functional, physiological or dynamic laws regulating the behaviour of the substratum.

It is suggested that in the central nervous system the stimuli enter and work themselves out as a pattern of nerve impulses on the cortex or gray matter generally, and that they are dependent on the cortex only in so far as the cortex provides a suitable medium on which the pattern can move and develop. According to such a hypothesis, a visual pattern thrown on to the visual cortex would not lie there as a static, fixed pattern, nor would it be projected en bloc to some other part of the cortex, but it. would immedtately 
grow, spread and irradiate over the whole cortex, just like the ripples spreading over the pond. Now there is in this connexion a well-established experimental fact, which shows that stimuli do in fact tend to spread over the cortex in this manner. I refer to the phenomenon of 'irradiation' described by Pavlov ${ }^{1}$. He has shown that any stimulus which arrives at the cortex immediately begins to spread over the surrounding cortex. $\mathrm{He}^{13}$ is of the opinion that this phenomenon is of fundamental importance in the physiology of the cerebral cortex. Now according to the path theory, this phenomenon has no meaning whatsoever. In fact, such an occurrence in a system run on path lines would lead immediately to chaos. The path theory and the fact of 'irradiation' are, as far as one can see, mutually exclusive and contradictory. On the other hand, the phenomenon of irradiation and what we may call the 'pattern' or 'ripple' theory of cortical function, are mutually complementary. The 'pattern' theory needs irradiation in order that the pattern may develop, while if one is given irradiation, then one may draw the logical conclusion that if one impulse spreads, a group of impulses will also spread, and of course the result of the spreading will depend on the distribution of stimuli in the original pattern and on the nature of the medium over which it spreads.

Such a theory would fit the facts of the recognition of objects in different parts of the visual field. For just like the ripples in the pond of water, so would the pattern developed depend solely on the rhythm and distribution of the stimuli, and not at all on the place stimulated.

Lashley ${ }^{i, 8}$ has pointed out in this connexion that wherever the distribution of the stimuli in space is of importance, one will find a spatial projection of the fibres on the cortex. It is, of course, in vision above all where the distribution of the stimuli in space is of importance, and in the visual cortex we find an accurate projection of the retina. To take the opposite extreme, although high and low notes are recognised in music, yet this has no distinction in space and correspondingly we find no scalar projection of musical notes on the cortex. Lashley has pointed out that this fact has no meaning in the path theory. On the other hand, for the pattern theory it is of fundamental importance that it should be so, for the impulses or 'ripples' must be given a correct start if they are going to irradiate and spread to form a correct pattern.

The pattern theory would also clarify to some extent the present confusion regarding the exact position of the ' functional ' disorders of the nervous system. To return to the analogy of the pond and its ripples for a moment, we see that there are two separate and distinct ways in which an abnormal or unusual pattern may be produced. Firstly by changes in the underlying medium on which the ripples form - there might be a rock in the water, or the water might be patchy in temperature, or the whole water might change in viscosity, or there might be a log floating on the surface. All these abnormalities would be disturbances of the underlying medium and as such would naturally affect adversely any pattern developing on its surface. Such abnormalities would 
correspond to the abnormalities of behaviour produced in the living organism by such things as tumours, infections, toxins, etc. It may be noted that such an obstruction would interfere not with any given pattern (or reaction) but would tend to interfere most with the most complex patterns, and this, it is suggested, is similar to what is observed in organic cortical disturbances.

But there is a second type of disturbance which may be produced, viz., by abnormalities of the original stimuli which produce the pattern. Here the underlying medium, the water or the cortex, is perfectly normal, but it has abnormal or unusual stimuli thrown on it. The result, naturally, will be an abnormal pattern, but produced, not by any abnormality of the water (or cortex), but by the fact that the stimuli which started the pattern were abnormal. This, I would suggest, is a state of affairs comparable to the 'functional' disorders. There are thus two fundamental ways in which such a system may go wrong ; firstly because of an abnormality in the underlying medium, and secondly because of an abnormality in the stimuli producing the pattern. At this point I wish to introduce a proposition which would require separate consideration for its complete exposition. It is to the effect that the concept of 'psychogenesis' is equivalent to stating that the cause of abnormalities of behaviour lies in the fact that the individual is subjected to abnormal or unusual stimuli which are too conflicting with his previous experience to enable him to react adequately. Most psychogenesists are agreed, I believe, that the cause of abnormalities of behaviour lies in the environment; the latter can only influence the individual as stimuli. That this type of disorder exists has been shown clearly by Pavlov'1. He has produced severe 'functional' breakdowns in dogs by subjecting them to stimuli which were neither frightening, nor loud, nor dazzling nor in any way harmful, but which were violently pathogenic simply because they were selected to conflict strongly with previously established reflexes. Abnormalities of behaviour, therefore, may be produced both by abnormalities of the brain and by abnormalities of the stimuli to which it is subjected.

It will be obvious that, according to the pattern theory, not only is there no conflict between the physiogenic and psychogenic theories of abnormalities of behaviour, but that, on the contrary, two such branches of knowledge are necessary : one to explain what will happen if the underlying medium is abnormal, and the other to explain what will happen if unusual groups of stimuli are presented.

It is interesting to note that in the second type of disorder, due to abnormal stimuli, the cortex will receive the impulses and will then use its own, perfectly normal methods for dealing with them, just as, in the pond, the water will deal perfectly normally with any pattern thrown upon it. Wilson ${ }^{14}$ has pointed out that from the neurological aspect the hysteric uses normal mechanisms for the development of his symptoms, while the psychologists, especially the psychoanalysts, have equally recognised that in investigating the mechanisms 
of psychopathology they find nothing but normal physiological mechanisms, acting on abnormal environmental material.

The pattern theory has been elaborated chiefly from the facts of cortical function, but we may justly enquire whether it has anything to say about reflex action. In its outline the theory has little to say except this--that since it is practically certain that the mechanisms of the brain and spinal cord will turn out to be essentially the same, we must therefore draw the conclusion that if the path theory will not fit cortical function, then it must be rejected as an explanation of reflex function. It was for this reason that I pointed out above that there is no evidence for the assumed constancy of the path of impulses in reflex action. Bourguignon's ${ }^{15}$ work on chronaxy strongly suggests, in fact, that the afferent impulses in the reflexes also irradiate diffusely in the gray matter but they can only escape from the spinal cord and show themselves down that nerve which has the same chronaxy. We may therefore, I think, invert the usual proceeding, and draw from the cortex conclusions regarding the mechanisms of reflex action.

Any value which the theory proposed may possess lies not in the immediate results achieved, but in the fact that it clears the ground, that it supplies an alternative to the path theory, and that it provides a new orientation to the subject, for a new orientation to the subject is certainly needed.

\section{REFERENCES.}

1 Feaking, F., Reftex Action, 1930.

$\because$ BeLL. C., Idea of a new Anatomy of the Brain, 1811.

3 BeLL, C., The Nervous System of the Human Body, 1833.

${ }^{4}$ HaLL, M., Synopsis of the Diastaltic Nerrous System, 1850.

5 Lewes, G. H., The Physical Basis of Mind, 1867.

' Herrick, C. J., Brains of Rats and Men, 1926.

' LAShley, K. S., "Basic neural mechanisms in behaviour," Psychol. Rer., 1930, xxxvii, 1.

${ }^{8} \mathrm{LASHLEY}, \mathrm{K}$. S., Brain Mechanisms and Intelligence, 1929.

${ }^{9}$ LASHLEY, K. S., and Franz, S. I., " The effects of cerebral destruction on habit formation and retention in the albino rat," Psychobiology, 1917, i, 71.

10 Lashley, K. S., Jour. Comp. Neurol., 1926, xli, 1.

11 Pavlov, I. P., Conditioned Reflexes, 1927.

1. Marina, A., Neurol. Centralbl., 1915, xxxiv, 338.

${ }^{13}$ Pavlov, I. P., " La vraie physiologie cérébrale," Arch. internat. ae physiol., 1921, xvili, 607.

14 Wilsox, S. A. K., Proc. Roy. Soc. Med., 1930, xxiv (Sect. Psychiat., 1).

15 Botractigrox, G., "La chronaxie," Nouveau traité de medécine, xviii, 521. 